\title{
A BINOMIALLY COEFFICIENT CLASS OF INTEGRATORS
}

\author{
Aashikpelokhai, U.S.U, Elakhe, A.O and Onianwa, C.U
}

Abstract - A portable class of integrators whose numerator and denominator coefficients are Binomial in form is derived. The derivation is based on a known stability function of the arbitrary $[\mathrm{L}, \mathrm{M}]$ rational integrator,

$$
y_{n+1}=\left[\sum_{r=0}^{L} p_{r} x_{n+1}^{r}\right]\left[1+\sum_{r=1}^{M} q_{r} x_{n+1}^{r}\right]^{-1}
$$

where $\mathrm{L}$ and $\mathrm{M}$ are the degrees of the respective numerator and denominator polynomials and

$$
p_{r}=\sum_{\alpha=0}^{\mathrm{r}} \frac{\mathrm{h}^{\alpha} y_{n}^{(\alpha)}}{\alpha ! x_{n+1}^{\alpha}} q_{r-\alpha}, \quad \mathrm{r}=0(1) \mathrm{L}
$$

$\left[\mathrm{q}_{\mathrm{m}}, \mathrm{q}_{\mathrm{m}-1}, \ldots . \mathrm{q}_{2}, \mathrm{q}_{1}\right]$ are obtained from the simultaneous linear algebraic equations $\mathbf{A q}=\mathbf{b}$

such that,

$\mathbf{A}=\left[\mathrm{a}_{\mathrm{ij}}\right]$ with $\mathrm{a}_{\mathrm{ij}}=\frac{\mathrm{h}^{\gamma} y_{n}^{(\gamma)}}{\gamma ! x_{n+1}^{\gamma}}$ and $\gamma=\mathrm{i}+\mathrm{j}+\mathrm{L}-\mathrm{M}-1$

$\mathbf{q}=\left[\mathrm{q}_{\mathrm{m}}, \mathrm{q}_{\mathrm{m}-1}, \ldots . ., \mathrm{q}_{2}, \mathrm{q}_{1}\right]$

$\mathbf{b}=\left[\mathrm{b}_{\mathrm{i}}\right]$ with $\mathrm{b}_{\mathrm{i}}=-\frac{\mathrm{h}^{\beta} y_{n}^{(\beta)}}{\beta ! x_{n+1}^{\beta}}$ and $\beta=\mathrm{L}+\mathrm{i}$

The stability function of this rational integrator is obtained and through which we obtain our initial Binomially Coefficient integrators. The research went further to prove the convergence and consistency of the derived integrators. Experiments carried out show good encouragement in the applications.

Keywords: Binomially Coefficient, Rational Integrators, Consistent, Convergent.

\section{Introduction}

The object of our study is the stability function of a general rational integrator reported in Aashikpelokhai (1991) and whose underlying interpolant is a rational function $\mathrm{P}_{\mathrm{L}}(\mathrm{x}) \mathrm{Q}_{\mathrm{M}}(\mathrm{x})^{-1}$ where $\mathrm{P}_{\mathrm{L}}(\mathrm{x}), \mathrm{Q}_{\mathrm{M}}(\mathrm{x})$ are polynomial function of degrees $L$ and $M$ respectively.

The stability function of any integrator is what is normally used in determining the Region of Absolute Stability of such an integrator. A common yardstick which is used in the determination of the Region of Absolute Stability (RAS) is the unit ball in $\mathrm{R}^{\mathrm{n}}$. The RAS is the same as the region in which the absolute value of the stability function lies in the unit ball in $\mathrm{R}^{\mathrm{n}}$.

Our computational experience as exemplified by the works in Aashikpelokhai (1991, 2000), Fatunla and Aashikpelokhai (1994), Elakhe and Aashikpelokhai (2013) along with the research work given by Fatunla (1982), Lambert and Shaw (1965), Otunta and Ikhile (1996, 1999) all give credence to the need for rational integrators

Aashikpelokhai, U.S.U ${ }^{1}$ and Elakhe, A.O ${ }^{2}$ (Authors)

Department of Mathematics, Ambrose Alli University

Ekpoma, Edo State, Nigeria

Onianwa, C.U(Author)

Department of Computer Sciences, Ambrose Alli University Ekpoma, Edo State, Nigeria despite the difficulties and unwieldy hard times rational integrators subject practitioners into.

To avoid the unwieldy hard times, one would want to know if it is possible to have some solace from the stability functions of known integrators by way of developing new integrators from them. If new integrators can be developed from them what advantage do we hope to enjoy from the new integrators? - (i) easy implementation, (ii) portability of such new methods. Do the new methods in turn sacrifice anything? - yes. They sacrifice high accuracies enjoyed by the parent rational integrators. To what extent is the sacrifice? This raises the question of the possibility of certain distinct integrators having identical stability functions and if not, then, there must be one-to-one and onto correspondence between all existing integrators and all stability functions. Our computational experience so far shows no such one-to-one and onto correspondence. The truth of this non-uniqueness is amptly demonstrated by the results stated in the table hereunder.

Table 1: The essential highlight of distinct integrators

\begin{tabular}{|c|c|c|}
\hline Numerical Integrator & Sources & \begin{tabular}{|c|} 
Stability \\
Functions
\end{tabular} \\
\hline$y_{n+1}=y_{n}+\frac{2 h f_{n}^{2}}{2 f_{2}-h f_{n}^{(1)}}$ & $\begin{array}{l}\text { Lambert } \\
(1974) \\
\text { Two } \\
\text { unconventi } \\
\text { onal } \\
\text { classes of } \\
\text { methods } \\
\text { for stiff } \\
\text { systems } \\
\text { (Ed Willoughb } \\
\text { y, R.A) } \\
\text { Wilberd, } \\
\text { Germany } \\
\text { (171-186) }\end{array}$ & $\frac{2+\bar{h}}{2-\bar{h}}$ \\
\hline 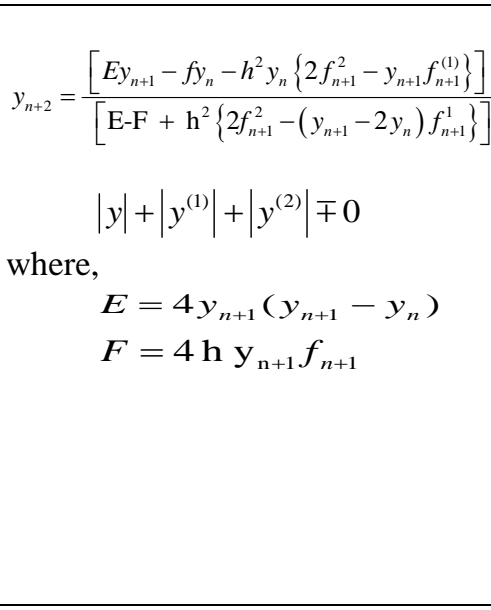 & $\begin{array}{l}\text { Lambert } \\
\text { (1974) } \\
\text { Two } \\
\text { unconventi } \\
\text { onal } \\
\text { classes of } \\
\text { methods } \\
\text { for stiff } \\
\text { systems } \\
\text { (Ed } \\
\text { Willoughb } \\
\text { y, R.A) } \\
\text { Wilberd, } \\
\text { Germany } \\
\text { (171-186) }\end{array}$ & $\frac{2+\bar{h}}{2-\bar{h}}$ \\
\hline$y_{n+1}=y_{n}+\frac{h}{2}\left[f_{n+1}+f_{n}\right]$ & $\begin{array}{l}\text { Trapezoidal } \\
\text { Rule }\end{array}$ & $\frac{2+\bar{h}}{2-\bar{h}}$ \\
\hline
\end{tabular}
having the same stability function. 
The concern at this stage is to provide an answer to a natural question arising from the facts shown in the above the table. The question reads: Given a particular stability function, can one derive or construct a numerical integrator from the given stability function despite the distinctness or uniqueness in the different integrators that exist and gave rise to it? Following the question is the next concern, namely: If such an integrator can be found, are there desirable properties possessed by it to give us hope of good performance? The answer to the first question is the main focus of this paper and so the answers and what lead to them are given in the next sections. For the second question we use the theories in Lambert $(1973,1976,1995)$ to determine the suitability of integrators for practical uses while the work in Enright and Pryce (1987) provides us with facilities for testing the suitability of numerical integrators for practical uses.

\section{Definition (Consistency)}

A one-step numerical integrator is said to be consistent if the integrator expression (potential function) represented by $\frac{y_{n+1}-y_{n}}{h}$ tends to $y_{n}^{(1)}$ as h tends to zero.

\section{Theorem (Consistency and Convergence)}

A one-step numerical integrator is consistent if and only if it is convergent (Lambert $(1976,1995))$.

To carry out our investigation, we would use the usual test equation,

$$
y^{(1)}=\lambda y
$$

where $\lambda$ represents an arbitrary eigenvalue of the differential system

$$
y^{(1)}=\mathbf{f}(x, \mathbf{y}), \mathbf{y}\left(x_{0}\right)=\mathbf{y}_{0}, \mathrm{x}_{0} \leq \mathrm{x} \leq \mathrm{x}_{\mathrm{f}}
$$

The usual eigenvalue - meshsize relation needed is

$$
\bar{h}=\lambda \mathrm{h}
$$

\section{The Binomially Coefficient Integrators}

Given two arbitrary nonnegative integers L, M, an integrator is defineable by,

where

$$
U(x)=\mathrm{P}_{\mathrm{L}}(x) \mathrm{Q}_{\mathrm{M}}^{-1}(x)
$$

$$
\begin{aligned}
& P_{L}(x)=\sum_{\mathrm{r}=0}^{\mathrm{L}} \mathrm{p}_{\mathrm{r}} x^{r} \\
& Q_{M}(x)=\sum_{\mathrm{r}=0}^{\mathrm{M}} \mathrm{q}_{\mathrm{r}} x^{r}, \quad \mathrm{Q}_{\mathrm{M}}(0) \equiv 1
\end{aligned}
$$

are real polynomial of degrees L, M respectively; subject to the integrator constraints,

$$
U\left(x_{n+i}\right)=\left\{\begin{array}{l}
y_{n+i}, \quad \mathrm{i}=0,1 \\
\mathrm{y}\left(\mathrm{x}_{\mathrm{n}+\mathrm{i}}\right) \text { for } \mathrm{i}=0 \text { only }
\end{array}\right.
$$

The resulting general rational integrator is,

$$
y_{n+1}=\left[\sum_{r=0}^{L} p_{r} x_{n+1}^{r}\right]\left[1+\sum_{r=1}^{M} q_{r} x_{n+1}^{r}\right]^{-1}
$$

where

$$
p_{r}=\sum_{\alpha=0}^{\mathrm{r}} \frac{\mathrm{h}^{\alpha} y_{n}^{(\alpha)}}{\alpha ! x_{n+1}^{\alpha}} q_{r-\alpha}, \quad \mathrm{r}=0(1) \mathrm{L}
$$

$\left[\mathrm{q}_{\mathrm{m}}, \mathrm{q}_{\mathrm{m}-1}, . . . \mathrm{q}_{2}, \mathrm{q}_{1}\right]$ are obtained from the simultaneous linear algebraic equations

$$
\mathbf{A q}=\mathbf{b}
$$

such that,

$$
\begin{aligned}
& \mathbf{A}=\left[\mathrm{a}_{\mathrm{ij}}\right] \text { with } \mathrm{a}_{\mathrm{ij}}=\frac{\mathrm{h}^{\gamma} y_{n}^{(\gamma)}}{\gamma ! x_{n+1}^{\gamma}} \text { and } \gamma=\mathrm{i}+\mathrm{j}+\mathrm{L}-\mathrm{M}-1 \\
& \mathbf{q}=\left[\mathrm{q}_{\mathrm{m}}, \mathrm{q}_{\mathrm{m}-1}, \cdot . \cdot, \mathrm{q}_{2}, \mathrm{q}_{1}\right] \\
& \mathbf{b}=\left[\mathrm{b}_{\mathrm{i}}\right] \text { with } \mathrm{b}_{\mathrm{i}}=-\frac{\mathrm{h}^{\beta} y_{n}^{(\beta)}}{\beta ! x_{n+1}^{\beta}} \text { and } \beta=\mathrm{L}+\mathrm{i}
\end{aligned}
$$

The stability function of this rational integrator is,

$$
S(\bar{h})=\frac{y_{n+1}(\bar{h})}{y_{n}(\bar{h})}=\frac{\sum_{r=0}^{L}(L+M-r) !\left(\begin{array}{l}
L \\
r
\end{array}\right) \bar{h}^{r}}{\sum_{r=0}^{M}(-1)^{r}(L+M-r) !\left(\begin{array}{l}
M \\
r
\end{array}\right) \bar{h}^{r}}
$$

where the expression: $\left(\begin{array}{l}k \\ \alpha\end{array}\right)=\frac{k !}{\alpha !(k-\alpha) !}$ for any pair of natural number $\mathrm{k}$ and $\alpha . \quad k-\alpha \geq 0$

For $r=0,1,2, . .$. , the relation (1) implies that at the integration point (ie at $\mathrm{x}=\mathrm{x}_{\mathrm{n}}$ ) we may write,

$$
y_{n}^{(r)}=\lambda^{\mathrm{r}} y_{n}
$$

and that for a k-step length, at the integration point, ( ie at $\mathrm{x}$ $=\mathrm{x}_{\mathrm{n}+\mathrm{k}}=\mathrm{x}_{\mathrm{n}}+\mathrm{kh}$ ) the relation (1) may be replaced by,

$$
y_{n+k}^{(r)}=\lambda^{\mathrm{r}} y_{n+k}
$$

Consequently, for each $\mathrm{r}=0,1,2$, . .

$$
(\bar{h})^{r}=\mathrm{h}^{\mathrm{r}} \lambda^{r}=\frac{\mathrm{h}^{\mathrm{r}} y_{n+k}^{(r)}}{y_{n+k}}
$$

By marrying (14) and (17) we obtain our initial Binomially coefficient integrators,

Binomially

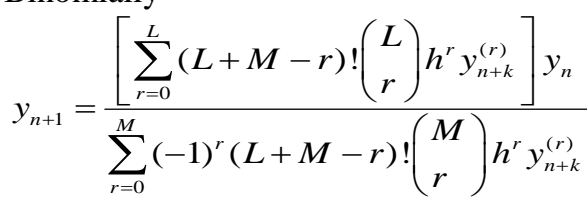

However, by virtue of the left - hand - side of (18), k cannot take any value distinct from 0 or 1 . Consequently, we write $\mathrm{k}=0,1$. The case $\mathrm{k}=0$ represents the explicit integrators while $\mathrm{k}=1$ represents the implicit integrators.

\section{Theorem 1 (Consistency and Convergence)}

The Binomially Coefficient class of integrators (18) are consistent and convergent if and only if they are explicit.

\section{Proof}

There are three mutually exclusive forms the integrator (18) assume; that is only one form at a time. The forms are $\mathrm{L}<$ $\mathrm{M}, \mathrm{L}=\mathrm{M}$ and $\mathrm{L}>\mathrm{M}$. We carry out our proof on a form by form basis.

\section{The form $L<M$}

The coefficients are $\left[\mathrm{p}_{\mathrm{r}} ; \mathrm{r}=0,1,2, \ldots, \mathrm{L}\right]$ and $\left[\mathrm{q}_{\mathrm{r}} ; \mathrm{r}=0,1,2\right.$, $\ldots, \mathrm{L}, \mathrm{L}+1, \mathrm{~L}+2, \ldots, \mathrm{M}]$ 
Proc. of the Fifth Intl. Conf. Advances in Computing, Communication and Information Technology- CCIT 2017 Copyright (C) Institute of Research Engineers and Doctors, USA .All rights reserved.

The integrators (18) may therefore be re-written as:

$$
y_{n+1}-y_{n}=\frac{\left[\begin{array}{l}
\left.\sum_{r=0}^{L}(L+M-r) !\left(\begin{array}{l}
L \\
r
\end{array}\right)-(-1)^{r}\left(\begin{array}{l}
M \\
r
\end{array}\right)\right\} h^{r} y_{n+k}^{(r)}- \\
\sum_{r=L}^{M}(-1)^{r}(L+M-r) !\left(\begin{array}{l}
M \\
r
\end{array}\right) h^{r} y_{n+K}^{(r)}
\end{array}\right] y_{n}}{\sum_{r=0}^{M}(-1)^{r}(L+M-r) !\left(\begin{array}{l}
M \\
r
\end{array}\right) h^{r} y_{n+k}^{(r)}}, \mathrm{k}=0,1
$$

The numerator becomes,

$$
\begin{aligned}
& {\left[(L+M) !\left\{\left(\begin{array}{l}
L \\
o
\end{array}\right)-\left(\begin{array}{l}
M \\
o
\end{array}\right)\right\} y_{n+k}+(L+M-1) !\left\{\left(\begin{array}{l}
L \\
1
\end{array}\right)+\left(\begin{array}{l}
M \\
1
\end{array}\right)\right\} h \mathrm{y}_{\mathrm{n}+\mathrm{k}}^{(1)}+\right.} \\
& \sum_{r=2}^{L}(L+M-r) !\left\{\left(\begin{array}{l}
L \\
r
\end{array}\right)-(-1)^{r}\left(\begin{array}{l}
M \\
r
\end{array}\right)\right\} h^{r} y_{n+k}^{(r)}- \\
& \left.\sum_{r=L+1}^{M}(-1)^{r}(L+M-r) !\left(\begin{array}{l}
M \\
r
\end{array}\right) h^{r} y_{n+k}^{(r)}\right] y_{n} \\
& {\left[(L+M-1) !(L+M) h \mathrm{y}_{\mathrm{n}+\mathrm{k}}^{(1)}+\right.} \\
& =\quad \sum_{r=2}^{L}(L+M-r) !\left\{\left(\begin{array}{l}
L \\
r
\end{array}\right)-(-1)^{r}\left(\begin{array}{l}
M \\
r
\end{array}\right)\right\} h^{r} y_{n+k}^{(r)}- \\
& \left.\sum_{r=L+1}^{M}(-1)^{r}(L+M-r) !\left(\begin{array}{l}
M \\
r
\end{array}\right) h^{r} y_{n+k}^{(r)}\right] y_{n}
\end{aligned}
$$$$
=
$$

$$
\begin{aligned}
& {\left[(L+M) ! h \mathrm{y}_{\mathrm{n}+\mathrm{k}}^{(1)}+\sum_{r=2}^{L}(L+M-r) !\left(\begin{array}{l}
L \\
r
\end{array}\right)-(-1)^{r}\left(\begin{array}{l}
M \\
r
\end{array}\right)\right\} h^{r} y_{n+k}^{(r)}-} \\
& \left.\sum_{r=L+1}^{M}(-1)^{r}(L+M-r) !\left(\begin{array}{l}
M \\
r
\end{array}\right) h^{r} y_{n+k}^{(r)}\right] y_{n}
\end{aligned}
$$$$
=h \mathrm{y}_{\mathrm{n}} \beta_{1}\left(h ; \mathrm{x}_{\mathrm{n}}, y_{n+k}\right), \mathrm{k}=0,1
$$

where

$$
\begin{gathered}
\beta_{1}\left(h ; \mathrm{x}_{\mathrm{n}}, y_{n+k}\right) \\
=(L+M) ! \mathrm{y}_{\mathrm{n}+\mathrm{k}}^{(1)}+\sum_{r=2}^{L}(L+M-r) !\left\{\left(\begin{array}{l}
L \\
r
\end{array}\right)-(-1)^{r}\left(\begin{array}{l}
M \\
r
\end{array}\right)\right\} h^{r-1} y_{n+k}^{(r)} \\
-\sum_{r=L+1}^{M}(-1)^{r}(L+M-r) !\left(\begin{array}{l}
M \\
r
\end{array}\right) h^{r-1} y_{n+k}^{(r)}, \mathrm{k}=0,1
\end{gathered}
$$

We denote the denominator by re-writing it as,

$\beta_{2}\left(h ; \mathrm{x}_{\mathrm{n}}, y_{n+k}\right)=$

$(L+M) ! \mathrm{y}_{\mathrm{n}+\mathrm{k}}+\sum_{r=1}^{M}(-1)^{r}(L+M-r) !\left(\begin{array}{l}M \\ r\end{array}\right) h^{r} y_{n+k}^{(r)}, k=0,1$

By these notations, the Binomially Coefficient class of integrator becomes,

$$
\begin{gathered}
y_{n+1}-y_{n}= \\
h \mathrm{y}_{\mathrm{n}} \beta_{1}\left(h ; \mathrm{x}_{\mathrm{n}}, y_{n+k}\right) \beta_{2}^{-1}\left(h ; \mathrm{x}_{\mathrm{n}}, y_{n+k}\right), \mathrm{k}=0,1 \\
=\quad h \theta\left(h ; \mathrm{x}_{\mathrm{n}}, y_{n+k}\right)
\end{gathered}
$$

where,

$$
\begin{aligned}
& \theta\left(h ; \mathrm{x}_{\mathrm{n}}, y_{n+k}\right)= \\
& \mathrm{y}_{\mathrm{n}} \beta_{1}\left(h ; \mathrm{x}_{\mathrm{n}}, y_{n+k}\right) \beta_{2}^{-1}\left(h ; \mathrm{x}_{\mathrm{n}}, y_{n+k}\right), \mathrm{k}=0,1
\end{aligned}
$$

One can now, write the Binomially Coefficient integrator as,

$$
y_{n+1}=y_{n}+h \theta\left(h ; \mathrm{x}_{\mathrm{n}}, y_{n+k}\right), \mathrm{k}=0
$$

Hence $\theta\left(h ; \mathrm{x}_{\mathrm{n}}, y_{n+k}\right)$ is the potential function of the integrator.

Consider $\operatorname{Limit}_{h \rightarrow 0} \beta_{1}\left(h ; \mathrm{x}_{\mathrm{n}}, y_{n+k}\right)=(\mathrm{L}+\mathrm{M}) ! \mathrm{y}_{\mathrm{n}+\mathrm{k}}^{(1)}$ and

$$
\operatorname{Limit}_{h \rightarrow 0} \beta_{2}\left(h ; \mathrm{x}_{\mathrm{n}}, y_{n+k}\right)=(\mathrm{L}+\mathrm{M}) ! \mathrm{y}_{\mathrm{n}+\mathrm{k}}
$$

implying

$$
\begin{aligned}
\theta\left(0 ; \mathrm{x}_{\mathrm{n}}, y_{n+k}\right) & =\operatorname{Limit}_{h \rightarrow 0} \theta\left(h ; \mathrm{x}_{\mathrm{n}}, y_{n+k}\right) \\
& =\frac{(L+M) ! y_{n+k}^{(1)} y_{n}}{(L+M) ! y_{n+k}} \\
& =\quad y_{n}^{(1)} \quad \text { if and only if } \mathrm{k}=0
\end{aligned}
$$

We conclude therefore that the Binomially Coefficient rational integrator (18) is consistent with the initial value problem if and only if it is explicit. By Lambert (1976, 1995) the explicit Binomially Coefficient integrator (18) is also convergent. This completes the proof for the form $\mathrm{L}<$ M.

\section{The form $L=M$}

By similar analysis carried out in the case $\mathrm{L}<\mathrm{M}$, we obtain here,

$$
\begin{aligned}
& \beta_{1}\left(h ; \mathrm{x}_{\mathrm{n}}, y_{n+k}\right)= \\
& (2 L) ! \mathrm{y}_{\mathrm{n}+\mathrm{k}}^{(1)}+\sum_{r=2}^{L}(2 L-r) !\left(\begin{array}{l}
L \\
r
\end{array}\right)\left\{1-(-1)^{r}\right\} h^{r-1} y_{n+k}, k=0,1 \\
& \beta_{2}\left(h ; \mathrm{x}_{\mathrm{n}}, y_{n+k}\right)= \\
& (2 L) ! \mathrm{y}_{\mathrm{n}+\mathrm{k}}+\sum_{r=1}^{L}(-1)^{r}(2 L-r) !\left(\begin{array}{l}
L \\
r
\end{array}\right) h^{r} y_{n+k}^{(r)}, k=0,1
\end{aligned}
$$

making the Binomially Coefficient integrator to be written as,

$$
y_{n+1}=y_{n}+h \theta\left(h ; \mathrm{x}_{\mathrm{n}}, y_{n+k}\right)
$$

where,

$$
\begin{aligned}
\theta\left(h ; \mathrm{x}_{\mathrm{n}}, y_{n+k}\right) & = \\
y_{n} & \beta_{1}\left(h ; \mathrm{x}_{\mathrm{n}}, y_{n+k}\right) \beta_{2}^{-1}\left(h ; \mathrm{x}_{\mathrm{n}}, y_{n+k}\right)
\end{aligned}
$$

leading us to $\theta\left(0 ; \mathrm{x}_{\mathrm{n}}, y_{n+k}\right)=y_{n}^{(1)}$ if and only if $\mathrm{k}=0$.

This establishes the result for the form $\mathrm{L}=\mathrm{M}$.

\section{The form $L>M$}

In this form, the coefficients are $\left[p_{r}, \mathrm{r}=0,1,2, \ldots, \mathrm{M}, \mathrm{M}+1, \mathrm{M}+2, \ldots, \mathrm{L}\right] \quad$ and $\left[q_{r}, \mathrm{r}=0,1,2, \ldots, \mathrm{M}\right]$

By similar analysis we used above, we obtain $\beta_{1}\left(h ; \mathrm{x}_{\mathrm{n}}, y_{n+k}\right)=$

$\left.(L+M) ! \mathrm{y}_{\mathrm{n}+\mathrm{k}}^{(1)}+\sum_{r=2}^{M}(L+M-r) !\left(\begin{array}{l}L \\ r\end{array}\right)-(-1)^{r}\left(\begin{array}{l}M \\ r\end{array}\right)\right\} h^{r} y_{n+k}^{(r)}$

$-\sum_{r=1}^{L}(L+M-r) !\left(\begin{array}{l}L \\ r\end{array}\right) h^{r-1} y_{n+k}^{(r)}, \mathrm{k}=0,1$ 
$\beta_{2}\left(h ; \mathrm{x}_{\mathrm{n}}, y_{n+k}\right)=(L+M) ! \mathrm{y}_{n+k}+$

$\sum_{r=1}^{M}(-1)^{r}(L+M-r) !\left(\begin{array}{l}M \\ r\end{array}\right) h^{r-1} y_{n+k}^{(r)}, \mathrm{k}=0,1$

giving rise to,

$$
\mathrm{y}_{\mathrm{n}+1}=y_{n}+h \theta\left(h ; \mathrm{x}_{\mathrm{n}}, y_{n+k}\right)
$$

with,

$$
\begin{aligned}
& \theta\left(h ; \mathrm{x}_{\mathrm{n}}, y_{n+k}\right)= \\
& y_{n} \beta_{1}\left(h ; \mathrm{x}_{\mathrm{n}}, y_{n+k}\right) y_{n} \beta_{2}^{-1}\left(h ; \mathrm{x}_{\mathrm{n}}, y_{n+k}\right), \mathrm{k}=0,1
\end{aligned}
$$

Which finally yield

$$
\theta\left(0 ; \mathrm{x}_{\mathrm{n}}, y_{n+k}\right)=y_{n}^{(1)} \text { if and only if } \mathrm{k}=0 .
$$

There are no other forms to consider. Hence the set of integrators (18) is consistent and convergent if and only if it is explicit. In this case the integrators are given by

$$
y_{n+1}=\frac{\left[\begin{array}{l}
\sum_{r=0}^{L}(L+M-r) !\left(\begin{array}{l}
L \\
r
\end{array}\right) h^{r} y_{n}^{(r)} \\
y^{\prime}
\end{array} y_{n}\right.}{\sum_{r=0}^{M}(-1)^{r}(L+M-r) !\left(\begin{array}{l}
M \\
r
\end{array}\right) h^{r} y_{n}^{(r)}}
$$

\section{Remarks}

Practitioners of integrators for ivp are fully aware that integrators that are not consistent are of little value in application. Consequently, the above has shown that only the explicit integrators (cases $\mathrm{k}=0$ ) are useful. Hence the useful integrators we derived here are given in (18).

\section{NOTE - WORTHY CONSIDERATIONS}

In this section, we highlight special features such that, when taken full note of become very useful when we consider applications. The first subsection of this section handles those special features, which reduce computational costs. They are stated in three classes. This subsection also paves the way to stability consideration, which along with singularity are given attention in subsection B.

\section{A. Special Features}

There are three classes we herein highlight very briefly as follows

Class 1: $\mathrm{L}$ is an arbitrary positive integer while $\mathrm{M}=0$. The integrators are given by

$$
y_{n+1}=\frac{\sum_{r=0}^{L}(L-r) !\left({ }_{r}^{L}\right) h^{r} y_{n}^{(r)}}{L !}=\sum_{r=0}^{L} \frac{h^{r} y_{n}^{(r)}}{r !}
$$

which is a truncated Taylor series.

Observe that for this class $\mathrm{L}=1$ produces Euler: $y_{n+1}=y_{n}+h y_{n}^{(1)}$

The corresponding stability function of the class is

$$
S(\bar{h})=\frac{\left.\sum_{r=0}^{L}(L-r) !{ }_{r}^{L}\right) \bar{h}^{r}}{L !}=\sum_{r=0}^{L} \frac{\bar{h}^{r}}{r !}
$$

Class 2: $\mathrm{L}=0$ while $\mathrm{M}$ is an arbitrary positive integer. The integrator class is the inverse polynomial integrators

$$
\begin{array}{r}
y_{n+1}=\frac{M ! y_{n}^{2}}{\left.\sum_{r=0}^{M}(-1)^{r}(M-r) !{ }_{r}^{M}\right) h^{r} y_{n}^{(r)}} \\
=\frac{y_{n}^{2}}{\sum_{r=0}^{M}(-1)^{r} \frac{h^{r}}{r !} y_{n}^{(r)}}
\end{array}
$$

which is a truncated alternating inverse Taylor series with numerator $y_{n}^{2}$. For this special class, $\mathrm{M}=0$ produces $y_{n+1}=\frac{y_{n}^{2}}{y_{n}-h y_{n}^{(1)}}$ which is Fatunla (1982) with $\mathrm{k}=1$.

The corresponding stability function of the class is the inverse polynomial function

$$
S(\bar{h})=\frac{M !}{\sum_{r=0}^{M}(-1)^{r}(M-r) !\left(_{r}^{M}\right) \bar{h}^{r}}=\frac{1}{\sum_{r=0}^{M}(-1)^{r} \frac{\bar{h}^{r}}{r !}}
$$

Class 3: $\mathrm{L}$ and $\mathrm{M}$ are related by sequences. This class could yield as many sequences of integrators as the researchers can define. However, we cite two examples. These are found in:

(a) Aashikpelokhai (1991) where $\mathrm{L}=\mathrm{k}-1$ and $\mathrm{M}=\mathrm{k}$. This group actually gave birth to this research.

(b) Otunta and Ikhile (1996) where $\mathrm{L}=\mathrm{k}-1$ with $\mathrm{l}=$ $0(1) \mathrm{k}$ and $\mathrm{M}=\mathrm{k}$. This group in turn is an extension of Aashikpelokhai (1991).

\section{Theorem 2 (The integrator SNAG)}

The Binomially coefficient class of rational integrators (28) are not suitable for ivp whose initial values pass through the origin except the cases $\mathrm{M}=0$.

\section{Proof}

Let the initial solutions of any given ivp $y^{(1)}=f(x, y), y\left(x_{0}\right)=y_{0}, a \leq x \leq b$ pass through the origin. In this case, the given ivp has its initial values $y_{0}=y\left(x_{0}\right) \quad$ reduce to $y_{0}=y(0)=0 ;$ thus $a=0$ in $a \leq x \leq b$.

Consequently, whenever $M \neq 0$, we set $\mathrm{n}=0$ in the integrator (28) above to obtain the following results.

Numerator $=$

$\left[\sum_{r=0}^{L}(L+M-r) !\left({ }_{r}^{L}\right) h^{r} y_{0}^{(r)}\right] y_{0}=0 \quad$ since $y_{0}=0$

Denominator 
Hence the solution $y_{1}=0$.

By induction step on $\mathrm{n}, y_{n+1}=0$ for every $\mathrm{n}$.

However, whenever $M \equiv 0$ the integrators belong to class $\mathbf{1}$ above and we have them given by the Taylor series

$$
y_{n+1}=\sum_{r=0}^{L} \frac{h^{r} y_{n}^{(r)}}{r !}
$$

which handle problems whose initial values pass through the origin.

The proof is complete.

\section{B. Singulo - Stiff Solvers}

One of our major goal in this research is to identify those integrators whose stability function $S(\bar{h})$ satisfy the requirement $\|S(\bar{h})\|<1$ in the entire left - half of the $\mathrm{U}-$ $\mathrm{V}$ complex plane where $\bar{h}=u+i v, i=\sqrt{-1}$. Such integrators are called stiff solvers. Since we are also interested in solving singular problems, we can justifiably state in this subsection that we are guided and motivated by our desire to work on singulo - stiff differential systems. Singulo - stiff ordinary differential equations are they that are either singular or stiff usually both. Let us recall from elementary mathematics that if $y_{n+1}=y_{n}+h \phi\left(x_{n}, y_{n} ; h\right)$ is a one - step method that solves the given ivp (2), and if the ivp has a singularity at a point $x^{*}$ in $\left\lfloor x_{0}, x_{f}\right\rfloor$ then $y_{n+1} \rightarrow \pm \infty$ as $x \rightarrow x^{*}$. For our set of integrators herein, if $M \neq 0$ then the integrator is purely properly rational. Consequently, $y_{n+1} \rightarrow \pm \infty$ if and only if $\sum_{r=0}^{M}(-1)^{r}(L+M-r) !\left({ }_{r}^{M}\right) h^{r} y_{0}^{(r)}=0$; meaning that the singularities of the ivp are simply picked up by $\sum_{r=0}^{M}(-1)^{r}(L+M-r) !\left({ }_{r}^{M}\right) h^{r} y_{0}^{(r)}$ as its zeros.

Hence this theoretical foundation makes this class of Binomially Coefficient integrators suitable for solving singular ivp when $M \neq 0$.

When handling ivps with stiff characteristics, the Region of Absolute Stabilty (RAS) of the one - step method in use is called to play. In simplest form, a one - step method is well equipped to solve stiff ivps if the RAS of the one - step method is at least the same as the left - half of the complex plane ie $R A S \supseteq\{\hat{h}: \operatorname{Re} \hat{h}<0\}$. One - step methods that enjoy this RAS characteristics are said to be A - Stable. These requirements will guide our choice of the pair [L, M].

Definition [Lambert (1991): A one - step numerical method is said to be $\mathrm{L}$ - stable if it is $\mathrm{A}$ - stable and, in addition, when applied to the test equation $y^{1}=\lambda y, \lambda$, a complex constant with $\operatorname{Re}(\lambda)<0$, it yields $y_{n+1}=R_{L, M}(h \lambda) y_{n}$ where $\underset{\operatorname{Re}(h \lambda) \rightarrow-\infty}{\operatorname{Limit}} R_{L, M}(h \lambda)=0$.

Definition [Ehle (1969)]: Let $R_{L, M}(w)=\frac{\sum_{r=0}^{L} p_{r} w^{r}}{1+\sum_{r=1}^{M} q_{r} w^{r}}$ be a rational approximation to the exponential function $\ell^{w}$, w a complex variable, then the rational approximant $R_{L, M}(w)$ is said to be

(a) A - acceptable if $\left|R_{L, M}(w)\right|<1$ whenever $\operatorname{Re}(w)<0$

(b) $\mathrm{A}_{0}$ - acceptable if $\left|R_{L, M}(w)\right|<1$ whenever w is real and negative.

(c) L - acceptable if it is A - acceptable and $\underset{\operatorname{Re}(w) \rightarrow-\infty}{\operatorname{Limit}}\left|R_{L, M}(w)\right|=0$

From here, the obvious conclusion was drawn that a numerical integrator is $\mathrm{A}-, \mathrm{A}_{0}-$ or $\mathrm{L}$ - stable according as $R_{L, M}(\bar{h})$ is $\mathrm{A}-, \mathrm{A}_{0}-$ or $\mathrm{L}-$ Acceptable.

\section{What links Acceptability with Stability?}

We must show that when $R_{L, M}(w)$ approximant underlying the ivp is applied replacing $y^{(r)}$ in the ivp derivatives with the exponential function, that the two $R_{L, M}(w)$ will be identical. To establish the RAS of any numerical integrator, the test equation normally applied $y^{1}=\lambda y$ results in $y_{n+1}=R_{L, M}(\bar{h}) y_{n}, \bar{h}=h \lambda$.

The stability function $S(\bar{h})$ is defined as the ratio $y_{n+1} / y_{n}$. Hence $S(\bar{h})=R_{L, M}(\bar{h})$. In this case $|S(\bar{h})|<1 \Leftrightarrow\left|R_{L, M}(\bar{h})\right|<1$. Warner et at (1978) proved that $R_{L, M}(\bar{h})$ is $\mathrm{A}-$ acceptable if and only if $M-2 \leq L \leq M$. Consequently, a rational [L,M] integrator is A - stable if and only if $M-2 \leq L \leq M$ Consequently, we concentrate our attention on these constrained values of $\mathrm{L}$ and $\mathrm{M}$ with the hope of re arresting attention in their direction for greater application in singulo - stiff ivps.

\section{Applications}

We have used in this section $\mathrm{a}(-\mathrm{b})=\mathrm{a} \times 10^{-\mathrm{b}}$.

Test Problem 1: $y^{(1)}=1+y^{2}, y(0)=1,0 \leq x \leq 1$

Theoretical solution: $\quad \mathrm{y}=\tan \left(\mathrm{x}+\frac{\pi}{4}\right)$

Problem Type: Solution is oscillatory and has singularity at $\mathrm{X}=\frac{\pi}{4}$.

Results are available in Table 7.1. We have used TOL $=10^{-2}$ $=\mathrm{h}_{0}$. 
Table 2: Showing an automatic switch in the open interval $(0.73,0.75)$

\begin{tabular}{|c|c|c|c|c|c|c|}
\hline $\begin{array}{c}\text { S/ } \\
\text { No }\end{array}$ & $\begin{array}{c}\text { Ord } \\
\text { er }\end{array}$ & s & $\mathbf{h}$ & $\mathbf{x}$ & $\begin{array}{c}\text { Actual } \\
\text { Error }\end{array}$ & $\begin{array}{c}\text { N } \\
\text { STEP }\end{array}$ \\
\hline 1 & 1 & 1.0 & 0.1 & 0.1 & $2.05(-4)$ & 1 \\
\hline 2 & 1 & 1.0 & 0.1 & 0.2 & $2.22(-4)$ & 20 \\
\hline 3 & 1 & 1.0 & 0.1 & 0.3 & $2.48(-4)$ & 30 \\
\hline 4 & 1 & 1.0 & 0.1 & 0.4 & $2.94(-4)$ & 40 \\
\hline 5 & 1 & 1.0 & 0.1 & 0.5 & $3.80(-4)$ & 50 \\
\hline 6 & 1 & 1.0 & 0.1 & 0.6 & $5.74(-4)$ & 60 \\
\hline 7 & 1 & 1.0 & 0.1 & 0.7 & $1.27(-3)$ & 70 \\
\hline 8 & 1 & 1.0 & 0.1 & 0.74 & $2.53(-4)$ & 74 \\
\hline 9 & 2 & 0.2826 & 0.0095 & 0.7495 & $2.22(-3)$ & 76 \\
\hline 10 & 2 & 0.3107 & 0.0079 & 0.7764 & $2.07(-3)$ & 80 \\
\hline 11 & 2 & 1.4422 & 0.7170 & 0.9863 & $3.92(-3)$ & 90 \\
\hline 12 & 2 & 1.3583 & 0.0337 & 1.0000 & $3.10(-4)$ & 91 \\
\hline
\end{tabular}

Test Problem 2:

$$
\begin{aligned}
& \mathrm{y}^{(1)}=\left[\begin{array}{cc}
-2000 & 1000 \\
1 & -1
\end{array}\right] \mathrm{y}+\left[\begin{array}{l}
1 \\
0
\end{array}\right], y(0)=\left[\begin{array}{l}
0 \\
0
\end{array}\right] .0 \\
& \text { Theoretical solution: } \\
& \mathrm{y}^{\mathrm{t}}=\left[\begin{array}{ll}
-4.9975 & -5.0025(-4) \\
2.994(-2) & -1.002(-3)
\end{array}\right]\left[\begin{array}{l}
\mathrm{e}^{\lambda_{1} t} \\
\mathrm{e}^{\lambda_{2} t}
\end{array}\right]+\left[\begin{array}{l}
1(-3) \\
1(-3)
\end{array}\right]
\end{aligned}
$$

where $\lambda_{1}=-2000.500125, \lambda_{2}=-0.499875$.

Problem Type: Stiff with stiffness factor $=4(-4)$

Table 3: Results are available in $\mathrm{e}_{1}, \mathrm{e}_{2}$

\begin{tabular}{|c|c|c|c|c|c|}
\hline $\mathbf{S}$ & $\mathbf{h}$ & $\mathbf{x}$ & $\mathbf{e}_{\mathbf{1}}$ & $\mathbf{e}_{\mathbf{2}}$ & $\begin{array}{c}\mathbf{N} \\
\text { STEPS }\end{array}$ \\
\hline 0.50 & 0.005 & 0.005 & $1.52(-4)$ & $2.30(-4)$ & 1 \\
\hline 1.00 & 0.005 & 0.003 & $3.57(-8)$ & $1.57(-8)$ & 5 \\
\hline 1.00 & 0.005 & 0.055 & $3.53(-8)$ & $1.56(-9)$ & 10 \\
\hline 1.00 & 0.005 & 0.080 & $3.49(-8)$ & $1.54(-9)$ & 15 \\
\hline 1.00 & 0.005 & 0.105 & $3.45(-8)$ & $1.53(-9)$ & 20 \\
\hline 1.00 & 0.005 & 0.130 & $3.41(-8)$ & $1.52(-9)$ & 25 \\
\hline 1.00 & 0.005 & 0.155 & $3.67(-8)$ & $1.51(-9)$ & 30 \\
\hline 1.00 & 0.005 & 0.180 & $3.32(-8)$ & $1.50(-9)$ & 35 \\
\hline 1.00 & 0.005 & 0.205 & $3.28(-8)$ & $1.49(-9)$ & 40 \\
\hline
\end{tabular}

Let $\mathrm{e}_{1}, \mathrm{e}_{2}$ represent the accumulated errors in component 1 and 2 respectively at each computational point. The first three integrators were not able within the specifications to do the work. The fourth method, an L-Stable method of order $p=3$ handled the problem well within the given specifications.

For the problem, TOL $=10^{-2}$. The integrator maintained $\mathrm{s}=1.0$ throughout the computation having begun with $\mathrm{s}=0.50$. At this only low value for $s$ we have $\mathrm{x}=0.005$ with $\mathrm{e}_{1}=1.52(-4)$ and $e_{2}=2.30(-6)$ being the maxima errors registered. The rest of the errors are given by $\left|\mathrm{e}_{1}\right|<10^{-7}$ and $\left|\mathrm{e}_{2}\right|<10^{-8}$ with $\mathrm{s}=1.00$. We have used in this section $\mathrm{a}(-\mathrm{b})=\mathrm{a} \times 10^{-\mathrm{b}}, \mathrm{N}_{\mathrm{f}}=$ Number of functional evaluations.

\section{Conclusion}

In this paper we have presented a new binomially class of rational integrator that clearly shows that through binomial method various integrators can be derived freely from know stability functions. Once a choice of a stability function has been made that satisfies need criteria for the solution of given problem, a suitable rational integrator can then be derived from it which can then be used to handle the problems The research takes into account the possibility of ill conditioning situation and suggested normal way of handling such. The treatments of singularities are also highlighted as the scheme takes into account the possibility of varying magnitudes of stiffness and initial value problem whose solutions are singular or oscillatory. Experiments carried out confirm the suitability of the scheme derived in solving these classes of problems. The scheme would therefore be a welcome relief for those who have no particular methods in mind except know stability function with large region of absolute stability and will therefore be found useful in the solution of problems arising from chemical kinetics, population model problems, nuclear

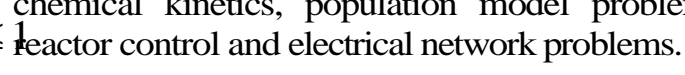

[1] Aashikpelokhai, U.S.U (1991): A Class of Non Linear One-Step Rational Integrator, Ph.D Uniben.

[2] Aashikpelokhai, U.S.U (2000): A variable order Numerical Integration Based on Rational Interpolats, JNMS 19 27-38.

[3] Fatunla S.O. and Aashikpelokhai, U.S.U (1994): A Fifth Order LStable Numerical Integrator Scientific Comp. 68-86.

[4] Elakhe, A. O. and Aashikpelokhai U.S.U (2013): Singulo Oscillatory - Stiff rational integrators. International Journal of Physical Sciences,

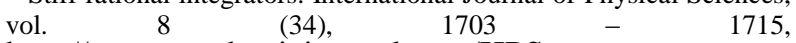
http://www.academicjournals.org/IJPS

[5] Fatunla, S.O. (1982): Non-Linear One Step Methods for ivp Comp. Method, Billic 18, 231-239.

[6] Lambert, J.D. \& Shaw, B (1965): On the Num Solution of $y^{(1)}=f(x, y)$ By a Clan of Forulae Based on Rational Interpolant Maths Comp. 19 $450-462$.

[7] Otunta, F.O \& Ikhile, M.N.O (1996): Stabilities of Convergence of a clan of variance Order Non-Linear One Step Rational Integrator of ivp in ODE. Int. J. Corp. Meth. 62, 49-61.

[8] Otunta, F.O \& Ikhile, M.N.O (1999): Efficient Rational One-step Num Integrators for ivp in ODE, J Coy Maths 72, 49-61.

[9] J. D. Lambert (1974): "Two Unconditional Classes of Methods for Stiff Systems (Ed Willoughby R.A) Wilbad W. Germany, 171-186.

[10] J.D. Lambert (1973): Computational Methods in Ordinary Differential Equations, John Wiley, New York.

[11] J. D. Lambert (1976): Convergence and Stability (Ed Hall G and Watt J.M, Oxford 20-44.

[12] Enright, W.H and Price J.D (1987): Two Fortran Packages for Assessing Initial Value Problems ACM Transactions a Math Software 13, 1-27.

[13] J. D. Lambert (1995): Numerical Methods for Ordinary Differential systems, John Wiley \& Sons Ltd., England

[14] J.D. Lambert (1991): Numerical Methods for Ordinary Differential System, John Wiley and Son, Chicharter Convergence.

[15] Ehle, B.L. (1969): On Padé approximations to the exponential function and $\mathrm{A}$ - stable methods for the numerical solution of initial value problems. University of Waterloo, Department of Applied Analysis and Computer Sciences, Research Report № CSRR2010. 